\title{
A search for the neutral Higgs boson at LEP
}

\section{L3 Collaboration}

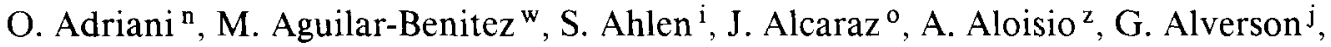
M.G. Alviggi ${ }^{\text {, G. Ambrosi }}{ }^{\text {ae }}$, Q. An ${ }^{\text {p }, ~ H . ~ A n d e r h u b ~}{ }^{\text {as }}$, A.L. Anderson ${ }^{\text {m }}$, V.P. Andreev ${ }^{\text {ai }}$, L. Antonov ${ }^{\mathrm{am}}$, D. Antreasyan ${ }^{\mathrm{g}}, \mathrm{P}$. Arce ${ }^{\mathrm{w}}, \mathrm{A}$. Arefiev ${ }^{\mathrm{y}}$, A. Atamanchuk ${ }^{\mathrm{ai}}$, T. Azemoon ${ }^{\mathrm{c}}$, T. Aziz ${ }^{\text {a,h }}$, P.V.K.S. Baba ${ }^{p}$, S. Bachmann ${ }^{a}$, P. Bagnaia ${ }^{\text {ah }}$, J.A. Bakken ${ }^{\text {ag }}$, L. Baksay $^{\text {ao }}$,

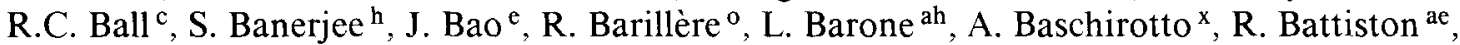
A. Bay ${ }^{\text {q }}$, F. Becattini ${ }^{n}$, U. Becker ${ }^{\mathrm{m} \text {,as }}$, F. Behner ${ }^{\text {as }}$, J. Behrens ${ }^{\text {as }}$, Gy.L. Bencze ${ }^{\mathrm{k}}$, J. Berdugo $^{\text {w }}$, P. Berges ${ }^{\mathrm{m}}$, B. Bertucci ${ }^{\text {ae }}$, B.L. Betev ${ }^{\text {am,as }}$, M. Biasini ${ }^{\text {ae }}$, A. Biland ${ }^{\text {as }}$, G.M. Bilei ${ }^{\text {ae }}$, R. Bizzarri ${ }^{\text {ah }}$, J.J. Blaising ${ }^{\mathrm{d}}$, G.J. Bobbink ${ }^{\mathrm{o}, \mathrm{b}}$, R. Bock ${ }^{\mathrm{a}}$, A. Böhm ${ }^{\mathrm{a}}$, B. Borgia ${ }^{\text {ah }}$, M. Bosetti ${ }^{\mathrm{x}}$, D. Bourilkov ${ }^{\text {ab }}$, M. Bourquin ${ }^{q}$, D. Boutigny ${ }^{d}$, B. Bouwens ${ }^{b}$, E. Brambilla ${ }^{z}$, J.G. Branson ${ }^{\text {aj }}$, I.C. Brock ${ }^{\text {af }}$, M. Brooks ${ }^{u}$, A. Bujak ${ }^{\text {ap }}$, J.D. Burger ${ }^{m}$, W.J. Burger ${ }^{q}$, J. Busenitz $^{\text {ao }}$, A. Buytenhuijs ${ }^{\text {ab }}$, X.D. Cai ${ }^{\mathrm{p}}$, M. Capell ${ }^{\mathrm{t}}$, M. Caria ${ }^{\text {ae }}$, G. Carlino $^{\mathrm{z}}$, A.M. Cartacci ${ }^{\mathrm{n}}$, R. Castello ${ }^{\mathrm{x}}$, M. Cerrada $^{\mathrm{w}}$, F. Cesaroni ${ }^{\text {ah }}$, Y.H. Chang ${ }^{\mathrm{m}}$, U.K. Chaturvedi ${ }^{\mathrm{p}}$, M. Chemarin ${ }^{\mathrm{v}}$, A. Chen ${ }^{\mathrm{au}}$, C. Chen ${ }^{\mathrm{f}}$, G.M. Chen ${ }^{\text {f }}$, H.F. Chen ${ }^{r}$, H.S. Chen ${ }^{\mathrm{f}}$, M. Chen ${ }^{\mathrm{m}}$, W.Y. Chen ${ }^{\mathrm{au}}$, G. Chiefari ${ }^{\mathrm{z}}$, C.Y. Chien ${ }^{\mathrm{e}}$, M.T. Choi ${ }^{\text {an }}$, S. Chung ${ }^{\mathrm{m}}$, C. Civinini ${ }^{n}{ }$, I. Clare ${ }^{\mathrm{m}}$, R. Clare ${ }^{\mathrm{m}}$, T.E. Coan ${ }^{\mathrm{u}}$, H.O. Cohn ${ }^{\mathrm{ac}}$,

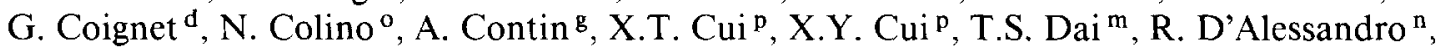
R. de Asmundis ${ }^{z}, A$. Degré ${ }^{d}, K$. Deiters ${ }^{\text {aq }}$, E. Dénes ${ }^{k}$, P. Denes ${ }^{\text {ag }}, F$. DeNotaristefani ${ }^{\text {ah }}$, M. Dhina ${ }^{\text {as }}$, D. DiBitonto ${ }^{a o}$, M. Diemoz ${ }^{\text {ah }}$, H.R. Dimitrov ${ }^{\text {am }}$, C. Dionisi ${ }^{\text {ah,o }}$, L. Djambazov ${ }^{\text {as }}$, M.T. Dova ${ }^{\text {p }}$, E. Drago ${ }^{z}$, D. Duchesneau ${ }^{\mathrm{q}}$, P. Duinker ${ }^{\mathrm{b}}$, I. Duran $^{\mathrm{ak}}$, S. Easo $^{\mathrm{ae}}$,

H. El Mamouni ${ }^{\text {v }}$, A. Engler af , F.J. Eppling ${ }^{\mathrm{m}}$, F.C. Erné ${ }^{\mathrm{b}}$, P. Extermann ${ }^{\mathrm{q}}$, R. Fabbretti ${ }^{\text {aq }}$, M. Fabre ${ }^{\mathrm{aq}}$, S. Falciano ${ }^{\text {ah }}$, S.J. Fan ${ }^{\mathrm{a} \ell}$, O. Fackler ${ }^{\mathrm{t}}$, J. Fay ${ }^{\mathrm{v}}$, M. Felcini ${ }^{\mathrm{o}}$, T. Ferguson ${ }^{\text {af }}$, D. Fernandez ${ }^{w}$, G. Fernandez ${ }^{w}$, F. Ferroni ${ }^{\text {ah }}$, H. Fesefeldt ${ }^{\text {a }}$, E. Fiandrini ${ }^{\text {ae }}$, J. Field ${ }^{q}$, F. Filthaut $^{\text {ab }}$, G. Finocchiaro ${ }^{\text {ah }}$, P.H. Fisher ${ }^{\mathrm{e}}$, G. Forconi ${ }^{\mathrm{q}}$, T. Foreman ${ }^{\mathrm{b}}$, K. Freudenreich ${ }^{\text {as }}$, W. Friebel ${ }^{\text {ar }}$, M. Fukushima ${ }^{\mathrm{m}}$, M. Gailloud ${ }^{\mathrm{s}}$, Yu. Galaktionov ${ }^{\mathrm{y}, \mathrm{m}}$, E. Gallo ${ }^{\mathrm{n}}$, S.N. Ganguli ${ }^{\mathrm{o}, \mathrm{h}}$, P. Garcia-Abia ${ }^{\text {w }}$, D. Gele ${ }^{v}$, S. Gentile ${ }^{\text {ah,o }}$, S. Goldfarb ${ }^{j}$, Z.F. Gong ${ }^{\text {, }}$, E. Gonzalez ${ }^{\text {w }}$,

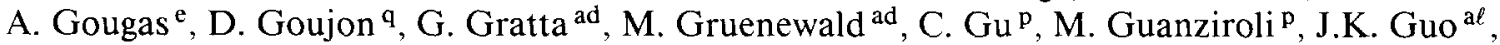
V.K. Gupta ${ }^{\text {ag }}$, A. Gurtu ${ }^{\text {h}}$, H.R. Gustafson ${ }^{\text {c }}$, L.J. Gutay ${ }^{\text {ap }}$, K. Hangarter ${ }^{\text {a }}$, A. Hasan ${ }^{\text {, }}$, D. Hauschildt ${ }^{b}$, C.F. He ${ }^{a \ell}$, J.T. He ${ }^{\mathrm{f}}$, T. Hebbeker ${ }^{\mathrm{a}}$, M. Hebert ${ }^{\text {aj }}$, G. Herten ${ }^{\mathrm{m}}$, A. Hervé ${ }^{\mathrm{o}}$,

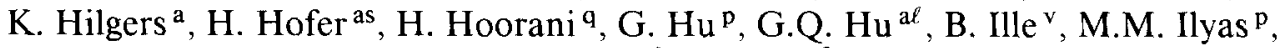
V. Innocente ${ }^{o}$, H. Janssen ${ }^{\circ}$, S. Jezequel ${ }^{d}$, B.N. Jin ${ }^{f}$, L.W. Jones ${ }^{c}$, A. Kasser ${ }^{s}$, R.A. Khan ${ }^{p}$, Yu. Kamyshkov ${ }^{a c}$, P. Kapinos ${ }^{a i, a r}$, J.S. Kapustinsky ${ }^{\text {a }}$, Y. Karyotakis ${ }^{\circ}$, M. Kaur ${ }^{\mathrm{p}}$, S. Khokhar $^{\mathrm{p}}$, M.N. Kienzle-Focacci ${ }^{\text {q }}$, J.K. Kim ${ }^{\text {an }}$, S.C. Kim ${ }^{\text {an }}$, Y.G. Kim ${ }^{\text {an }}$, W.W. Kinnison ${ }^{\text {u, D. Kirkby }}{ }^{\text {ad }}$, S. Kirsch ${ }^{\text {ar }}$, W. Kittel ${ }^{\text {ab }}$, A. Klimentov ${ }^{\text {m,y }}$, A.C. König ${ }^{\text {ab }}$, E. Koffeman ${ }^{b}$, O. Kornadt ${ }^{a}$, V. Koutsenko ${ }^{\mathrm{m}, \mathrm{y}}$, A. Koulbardis ${ }^{\mathrm{ai}}$, R.W. Kraemer ${ }^{\text {af }}$, T. Kramer ${ }^{\mathrm{m}}$, V.R. Krastev ${ }^{\text {am,ae }}$, W. Krenz ${ }^{\text {a }}$, A. Krivshich ${ }^{\text {ai }}$, H. Kuijten ${ }^{\text {ab }}$, K.S. Kumar ${ }^{\ell}$, A. Kunin ${ }^{\ell, y}$, G. Landi ${ }^{\text {n }}$, D. Lanske ${ }^{\text {a }}$, S. Lanzano ${ }^{\text {, }}$ P. Lebrun ${ }^{\vee}$, P. Lecomte ${ }^{\text {as }}$, P. Lecoq ${ }^{\circ}$, P. Le Coultre ${ }^{\text {as }}$, D.M. Lee ${ }^{\text {u }}$, I. Leedom $^{j}$, C. Leggett ${ }^{c}$, J.M. Le Goff ${ }^{o}$, R. Leiste ${ }^{\text {ar }}$, M. Lenti ${ }^{n}$, E. Leonardi ah, X. Leytens ${ }^{b}, C^{b}$ Li $^{r, p}$, H.T. Li ${ }^{\mathrm{f}}$, P.J. Li ${ }^{\mathrm{a} \ell}$, J.Y. Liao ${ }^{\mathrm{a} \ell}$, W.T. Lin ${ }^{\mathrm{au}}$, Z.Y. Lin ${ }^{\mathrm{r}}$, F.L. Linde ${ }^{\circ}$, B. Lindemann ${ }^{\mathrm{a}}$, L. Lista $^{\mathrm{z}}$, Y. Liu ${ }^{p}$, W. Lohmann ${ }^{\text {ar,o }}$, E. Longo ${ }^{\text {ah }}$, Y.S. Lu ${ }^{f}$, J.M. Lubbers $^{\circ}$, K. Lübelsmeyer ${ }^{a}$, C. Luci ${ }^{\text {h }}$, D. Luckey ${ }^{\text {g,m }}$, L. Ludovici ${ }^{\text {ah }}$, L. Luminari ${ }^{\text {ah }}$, W. Lustermann ${ }^{\text {ar }}$, J.M. Ma ${ }^{\text {f }}$, W.G. Ma ${ }^{\mathrm{r}}$, 
M. MacDermott ${ }^{\text {as }}$, P.K. Malhotra ${ }^{\text {h,3 }}$ R. Malik ${ }^{p}$, A. Malinin ${ }^{y}$, C. Maña ${ }^{w}$, M. Maolinbay as, P. Marchesini ${ }^{\text {as }}$, F. Marion ${ }^{\text {d, }}$ A. Marin ${ }^{\text {i }}$, J.P. Martin ${ }^{v}$, L. Martinez-Laso ${ }^{w}$, F. Marzano ${ }^{\text {ah }}$, G.G.G. Massaro $^{\text {b }}$, K. Mazumdar ${ }^{\text {h }}$, P. McBride ${ }^{\ell}$, T. McMahon ${ }^{\text {ap }}$, D. McNally ${ }^{\text {as }}$, M. Merk ${ }^{\text {af }}$, L. Merola ${ }^{z}$, M. Meschini ${ }^{n}$, W.J. Metzger ${ }^{\text {ab }}$, Y. Mi ${ }^{\text {s }, ~ G . B . ~ M i l l s ~}{ }^{\mathrm{u}}$, Y. Mir ${ }^{\mathrm{p}}$, G. Mirabelli $^{\text {ah }}$, J. Mnich ${ }^{a}$, M. Möller ${ }^{\text {a }}$, B. Monteleoni ${ }^{n}$, R. Morand ${ }^{\mathrm{d}}$, S. Morganti ${ }^{\text {ah }}$, N.E. Moulai ${ }^{p}$, R. Mount ${ }^{\text {ad }}$, S. Müller ${ }^{\text {a }}$, A. Nadtochy ${ }^{\text {ai }}$, E. Nagy ${ }^{\text {k }}$, M. Napolitano ${ }^{z}$, F. Nessi-Tedaldi as, H. Newman ${ }^{\text {ad }}$, C. Neyer ${ }^{\text {as }}$, M.A. Niaz ${ }^{\text {p }}$, A. Nippe ${ }^{\text {a }}$, H. Nowak ${ }^{\text {ar }}$, G. Organtini ${ }^{\text {ah }}$, D. Pandoulas ${ }^{\text {a }}$,

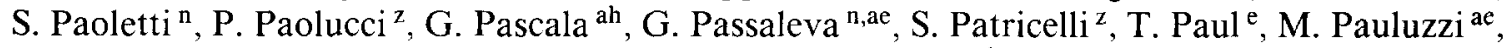
C. Paus ${ }^{\text {a }}$, F. Pauss ${ }^{\text {as }}$, Y.J. Pei ${ }^{a}$, S. Pensotti ${ }^{x}$, D. Perret-Gallix ${ }^{d}$, J. Perrier ${ }^{q}$, A. Pevsner ${ }^{e}$, D. Piccolo ${ }^{\mathrm{z}}$, M. Pieri ${ }^{\mathrm{o}}$, P.A. Piroué ${ }^{\mathrm{ag}}$, F. Plasil ${ }^{\mathrm{ac}}$, V. Plyaskin ${ }^{\mathrm{y}}, \mathrm{M}$. Pohl ${ }^{\text {as }}$, V. Pojidaev ${ }^{\mathrm{y}, \mathrm{n}}$, H. Postema ${ }^{\mathrm{m}}$, Z.D. Qi ${ }^{\mathrm{a} \ell}$, J.M. Qian $^{\mathrm{c}}$, K.N. Qureshi ${ }^{\mathrm{p}}$, R. Raghavan ${ }^{\mathrm{h}}$, G. Rahal-Callot ${ }^{\text {as }}$, P.G. Rancoita ${ }^{\mathrm{x}}$, M. Rattaggi ${ }^{x}$, G. Raven ${ }^{\mathrm{b}}$, P. Razis ${ }^{\text {aa }}$, K. Read ${ }^{\text {ac }}$, D. Ren ${ }^{\text {as }}$, Z. Ren $^{\mathrm{p}}$, M. Rescigno ${ }^{\text {ah }}$, S. Reucroft ${ }^{j}$, A. Ricker $^{a}$, S. Riemann ${ }^{\text {ar }}$, B.C. Riemers ${ }^{\text {ap }}$, K. Riles ${ }^{c}$, O. Rind ${ }^{c}$, H.A. Rizvi ${ }^{\text {p }}$, F.J. Rodriguez ${ }^{w}$, B.P. Roe ${ }^{c}$, M. Röhner ${ }^{\text {a }}$, S. Röhner ${ }^{\text {a }}$, L. Romero ${ }^{w}$, J. Rose ${ }^{\text {a }}$, S. Rosier-Lees ${ }^{d}$, R. Rosmalen ${ }^{\text {ab }}$, Ph. Rosselet ${ }^{\mathrm{s}}$, W. van Rossum ${ }^{\mathrm{b}}$, S. Roth ${ }^{\mathrm{a}}$, A. Rubbia ${ }^{\mathrm{m}}$, J.A. Rubio $^{\circ}$, H. Rykaczewski ${ }^{\text {as }}$, M. Sachwitz ${ }^{\text {ar }}$, J. Salicio ${ }^{\circ}$, J.M. Salicio ${ }^{\text {w }}$, G.S. Sanders ${ }^{\text {u, }}$ A. Santocchia ae M.S. Sarakinos ${ }^{\mathrm{m}}$, G. Sartorelli ${ }^{\text {a.p }}$, M. Sassowsky ${ }^{a}$, G. Sauvage ${ }^{d}$, C. Schäfer ${ }^{a}$, V. Schegelsky ai, D. Schmitz ${ }^{a}$, P. Schmitz ${ }^{a}$, M. Schneegans ${ }^{d}$, H. Schopper ${ }^{\text {at }}$, D.J. Schotanus ${ }^{\text {ab }}$, S. Shotkin ${ }^{\mathrm{m}}$, H.J. Schreiber ${ }^{\text {ar }}$, J. Shukla ${ }^{\text {af }}$, R. Schulte ${ }^{a}$, S. Schulte ${ }^{a}$, K. Schultze ${ }^{\text {a }}$, J. Schwenke ${ }^{\text {a }}$, G. Schwering ${ }^{\text {a }}$, C. Sciacca ${ }^{z}$, I. Scott ${ }^{\ell}$, R. Sehgal ${ }^{p}$, P.G. Seiler ${ }^{\text {aq }}$, J.C. Sens $^{\text {o,b }}$, L. Servoli $^{\text {ae }}$, I. Sheer ${ }^{\text {aj }}$, D.Z. Shen ${ }^{\text {a } \ell}$, S. Shevchenko ${ }^{\text {ad }}$, X.R. Shi ${ }^{\text {ad }}$, E. Shumilov ${ }^{y}$, V. Shoutko ${ }^{y}$, D. Son ${ }^{\text {an }}$, A. Sopczak ${ }^{\text {aj }}$, C. Spartiotis ${ }^{\text {e }}$, T. Spickermann ${ }^{\text {a }}$, P. Spillantini ${ }^{n}$, R. Starosta ${ }^{\text {a }}$, M. Steuer ${ }^{\text {g,m }}$, D.P. Stickland ${ }^{\text {ag }}$, F. Sticozzi ${ }^{\mathrm{m}}$, H. Stone ${ }^{\mathrm{ag}}$, K. Strauch ${ }^{\ell}$, B.C. Stringfellow ${ }^{\text {ap }}$, K. Sudhakar ${ }^{\text {h }}$, G. Sultanov $^{p}$, L.Z. Sun ${ }^{r, p}$, H. Suter ${ }^{\text {as }}$, J.D. Swain ${ }^{p}$, A.A. Syed ${ }^{a b}$, X.W. Tang ${ }^{f}$, L. Taylor ${ }^{j}$, G. Terzi ${ }^{x}$, Samuel C.C. Ting ${ }^{\mathrm{m}}$, S.M. Ting ${ }^{\mathrm{m}}$, M. Tonutti ${ }^{\mathrm{a}}$, S.C. Tonwar ${ }^{\mathrm{h}}$, J. Tóth $^{\mathrm{k}}$,

A. Tsaregorodtsev ${ }^{\text {ai }}$, G. Tsipolitis ${ }^{\text {af }}$, C. Tully ${ }^{\text {ag }}$, K.L. Tung ${ }^{f}$, J. Ulbricht ${ }^{\text {as }}$, L. Urbán ${ }^{k}$, U. Uwer $^{\mathrm{a}}$, E. Valente ${ }^{\mathrm{ah}}$, R.T. Van de Walle ${ }^{\mathrm{ab}}$, I. Vetlitsky $^{\mathrm{y}}$, G. Viertel $^{\text {as }}$, P. Vikas ${ }^{\mathrm{p}}$, U. Vikas ${ }^{\mathrm{p}}$, M. Vivargent ${ }^{d}$, H. Vogel ${ }^{\text {af }}$, H. Vogt ${ }^{\text {ar }}$, I. Vorobiev ${ }^{y}$, A.A. Vorobyov ${ }^{a i}$, L. Vuilleumier ${ }^{\text {s }}$, M. Wadhwa ${ }^{\mathrm{d}}$, W. Wallraff ${ }^{\mathrm{a}}$, C. Wang ${ }^{\mathrm{m}}$, C.R. Wang ${ }^{\mathrm{r}}$, G.H. Wang ${ }^{\text {af }}$, X.L. Wang ${ }^{\mathrm{r}}$, Y.F. Wang ${ }^{\mathrm{m}}$, Z.M. Wang ${ }^{\text {p,r }}$, A. Weber ${ }^{a}$, J. Weber ${ }^{\text {as }}$, R. Weill ${ }^{\mathrm{s}}$, T.J. Wenaus ${ }^{\mathrm{t}}$, J. Wenninger ${ }^{\mathrm{q}}$, M. White ${ }^{\mathrm{m}}$, C. Willmott ${ }^{w}$, F. Wittgenstein ${ }^{\circ}$, D. Wright ${ }^{\mathrm{ag}}$, S.X. Wu ${ }^{\mathrm{p}}, \mathrm{S}$. Wynhoff ${ }^{\mathrm{a}}$, B. Wystouch $^{\mathrm{m}}$, Y.Y. Xie ${ }^{a \ell}$, J.G. Xu ${ }^{\text {f }}, Z$ Z.Z. Xu ${ }^{r}$, Z.L. Xue ${ }^{a \ell}$, D.S. Yan ${ }^{a \ell}$, B.Z. Yang ${ }^{r}$, C.G. Yang ${ }^{f}$, G. Yang ${ }^{p}$,

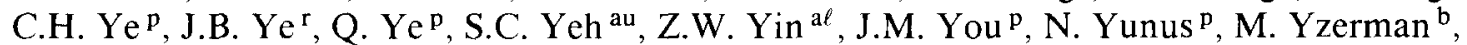
C. Zaccardelli ad , P. Zemp ${ }^{\text {as }}$, M. Zeng ${ }^{p}$, Y. Zeng ${ }^{a}$, D.H. Zhang ${ }^{b}$, Z.P. Zhang ${ }^{r, p}$, B. Zhou ${ }^{i}$, G.J. Zhou ${ }^{\text {f }}$,J.F. Zhou ${ }^{\text {a }, ~ R . Y . ~ Z h u ~}{ }^{\text {ad }}$, A. Zichichi ${ }^{\text {g,o,p }}$ and B.C.C. van der Zwaan ${ }^{b}$

a I. Physikalisches Institut, RWTH, W-5100 Aachen, FRG ${ }^{1}$; III. Physikalisches Institut, RWTH, W-5100 Aachen, FRG ${ }^{1}$

b National Institute for High Energy Physics, NIKHEF, NL-1009 DB Amsterdam. The Netherlands

c University of Michigan, Ann Arbor, MI 48109, USA

d Laboratoire d'Annecy-le-Vieux de Physique des Particules, LAPP, IN2P3-CNRS, BP 110, F-74941 Annecy-le-Vieux CEDEX, France

e Johns Hopkins University, Baltimore, MD 21218, USA

f Institute of High Energy Physics, IHEP, 100039 Beijing, China

g INFN-Sezione di Bologna, I-40126 Bologna, Italy

h Tata Institute of Fundamental Research, Bombay 400 005, India

i Boston University, Boston, MA 02215, USA

j Northeastern University, Boston, MA 02115, USA

k Central Research Institute for Physics of the Hungarian Academy of Sciences, H-1525 Budapest 114, Hungary ${ }^{2}$

' Harvard University, Cambridge, MA 02139. USA 
m Massachusetts Institute of Technology, Cambridge, MA 02139, USA

"INFN Sezione di Firenze and University of Florence, I-50125 Florence, Italy

- European Laboratory for Particle Physics, CERN, CH-1211 Geneva 23, Switzerland

p World Laboratory, FBLJA Project, CH-1211 Geneva 23, Switzerland

a University of Geneva, CH-1211 Geneva 4, Switzerland

${ }^{r}$ Chinese University of Science and Technology, USTC, Hefei, Anhui 230 029, China

s University of Lausanne, CH-1015 Lausanne, Switzerland

${ }^{t}$ Lawrence Livermore National Laboratory, Livermore, CA 94550, USA

"Los Alamos National Laboratory, Los Alamos, NM 87544, USA

$\checkmark$ Institut de Physique Nucléaire de Lyon, IN2P3-CNRS, Université Claude Bernard, F-69622 Villeurbanne Cedex, France

" Centro de Investigaciones Energeticas, Medioambientales y Tecnologicas, CIEMAT, E-28040 Madrid, Spain

x INFN-Sezione di Milano, I-20133 Milan, Italy

y Institute of Theoretical and Experimental Physics, ITEP, Moscow, Russia

${ }^{2}$ INFN-Sezione di Napoli and University of Naples, I-80125 Naples, Italy

aa Department of Natural Sciences, University of Cyprus, Nicosia, Cyprus

ab University of Nymegen and NIKHEF, NL-6525 ED Nymegen, The Netherlands

ac Oak Ridge National Laboratory, Oak Ridge, TN 37831, USA

ad California Institute of Technology, Pasadena, CA 91125, USA

ae INFN-Sezione di Perugia and Universitá Degli Studi di Perugia, I-06100 Perugia, Italy

af Carnegie Mellon University, Pittsburgh, PA 15213, USA

ag Princeton University, Princeton, NJ 08544, USA

ah INFN-Sezione di Roma and University of Rome, "La Sapienza", I-00185 Rome, Italy

ai Nuclear Physics Institute, St. Petersburg, Russia

aj University of California, San Diego, CA 92093, USA

ak Dept. de Fisica de Particulas Elementales, Univ. de Santiago, E-15706 Santiago de Compostela, Spain

al Shanghai Institute of Ceramics, SIC, Shanghai, China

am Bulgarian Academy of Sciences, Institute of Mechatronics, BU-1113 Sofia, Bulgaria

an Center for High Energy Physics, Korea Advanced Inst. of Sciences and Technology, 305-701 Taejon, Republic of Korea

ao University of Alabama, Tuscaloosa, AL 35486, USA

ap Purdue University, West Lafayette, IN 47907, USA

aq Paul Scherrer Institut, PSI, CH-5232 Villigen, Switzerland

ar DESY-Institut für Hochenergiephysik, O-1615 Zeuthen, FRG

as Eidgenössische Technische Hochschule, ETH Zürich. CH-8093 Zürich, Switzerland

at University of Hamburg, W-2000 Hamburg, FRG

au High Energy Physics Group. Taiwan, China

Received 18 February 1993

We update the results of a search for the Standard Model neutral Higgs boson using a data sample corresponding to 1062000 hadronic $Z$ decays. We exclude the existence of the Minimal Standard Model Higgs boson in the mass range $0 \leqslant m_{\mathrm{H}}<57.7 \mathrm{GeV}$ at the $95 \%$ confidence level.

\section{Introduction}

The Minimal Standard Model [1] predicts the existence of a neutral scalar Higgs particle $\mathrm{H}^{0}$ [2]. In

I Supported by the German Bundesministerium für Forschung und Technologie.

2 Supported by the Hungarian OTKA fund under contract number 2970.

3 Deceased. the framework of this model the couplings of the $\mathrm{H}^{0}$ boson to the fermions and to the gauge vector bosons are known but its mass is not specified.

The main production mechanism of the Higgs boson at LEP is predicted to be through the decay of the $\mathrm{Z}$ boson into an $\mathrm{H}^{0}$ and a virtual $\mathrm{Z}^{*}[3]$

$\mathrm{e}^{+} \mathrm{e}^{-} \rightarrow \mathrm{Z} \rightarrow \mathrm{H}^{0}+\mathrm{Z}^{*} \rightarrow \mathrm{H}^{0}+\mathrm{f} \overline{\mathrm{f}}$

A heavy Higgs boson (with mass exceeding $11 \mathrm{GeV}$ ) 
decays predominantly into a $b \bar{b}$ pair, although the branching ratios into $c \overline{\mathrm{c}}$ and $\tau^{+} \tau^{-}$are not negligible [4]. With QCD corrections [5] the branching ratio into $\tau^{+} \tau^{-}$for a $60 \mathrm{GeV}$ Higgs boson is approximately $5.7 \%$.

We have previously reported on searches for the Standard Model Higgs boson using the 1990 and 1991 data sample, corresponding to $17.5 \mathrm{pb}^{-1}$ integrated luminosity around the $Z$ pole $[6,7]$. These searches allowed us to exclude the presence of the Higgs boson in the mass range $0 \leqslant m_{\mathrm{H}}<52.0 \mathrm{GeV}$ at the $95 \%$ confidence level. Searches have also been performed by the other LEP experiments [8-10].

Using the same analysis method we have expanded the search for the $\mathrm{H}^{0}$ by including our 1992 data sample, corresponding to $654000 \mathrm{Z}$ hadronic decays. Altogether this totals 1062000 hadronic $Z$ decays and $39.0 \mathrm{pb}^{-1}$ integrated luminosity at center of mass energies between 88.2 and $94.3 \mathrm{GeV}$.

We report here on updated results of the search for the Higgs boson in the mass range from 30 to $70 \mathrm{GeV}$. We have searched in the channels $\mathrm{H}^{0} \nu \bar{\nu}, \mathrm{H}^{0} \mathrm{e}^{+} \mathrm{e}^{-}, \mathrm{H}^{0} \mu^{+} \mu^{-}$, where the signal has the most distinctive signatures.

\section{The $\mathbf{L 3}$ detector}

The L3 detector consists of a central tracking chamber, a high resolution electromagnetic calorimeter composed of $\mathrm{BGO}$ crystals, a ring of scintillation counters, a uranium and brass hadron calorimeter with proportional wire chamber readout, and an accurate muon chamber system. These detectors are installed in a $12 \mathrm{~m}$ diameter magnet which provides a uniform field of $0.5 \mathrm{~T}$ along the beam direction. For hadronic jets the fiducial coverage is $99 \%$ of $4 \pi$.

We have previously presented the detector and its performance in detail $[11,12]$. Here we briefly describe the subdetectors relevant for this analysis.

The central tracking chamber (TEC) is a time expansion chamber which consists of two cylindrical layers of 12 (inner) and 24 (outer) sectors, with 62 wires measuring the $r-\phi$ coordinate. The single wire resolution is $58 \mu \mathrm{m}$ averaged over the entire cell. The double-track resolution is $640 \mu \mathrm{m}$. The BGO electromagnetic calorimeter, which includes endcaps installed in 1991 , covers $85 \%$ of the solid angle. The fine segmentation of the BGO detector and hadron calorimeter allows us to measure the direction of jets with an angular resolution of $2.1^{\circ}$, and to measure the total energy of hadronic events from $Z$ decays with a resolution of $10.2 \%$. The muon detector consists of 3 layers of precise drift chambers, which measure 56 points on the muon trajectory in the bending plane, and 8 points in the non-bending direction.

The response of the detector was simulated using the $\mathrm{L} 3$ detector simulation program $[13,15]^{\# 1}$ which takes into account the effects of energy loss, multiple scattering, interactions and decays and includes the detector efficiency and resolution.

As the selections described below extensively use the information coming from the calorimetric part of the detector, we briefly describe the related reconstruction algorithm. Jets are reconstructed using a two step procedure [12]: firstly neighbouring calorimetric hits are combined into clusters, then jets are formed merging neighbouring clusters and muon tracks. Each charged track measured in the tracking chamber is assigned to the nearest jet. The algorithm normally reconstructs one such 'jet' for a single isolated electron, photon, muon, high energy $\tau$ or a hadronic jet. Unless otherwise stated in the following jets are defined by this algorithm.

\section{3. $\mathrm{H}^{0} \mathrm{v} \overline{\mathrm{v}}$ event selection}

$\mathrm{H}^{0} \nu \bar{\nu}$ events are characterized by large missing energy and momentum imbalance due to the undetected neutrinos from the $Z^{*}$ decay. The heavy quarks from the Higgs decay receive a Lorentz boost leading to two acoplanar jets which mainly populate one hemisphere with a rather low energy deposit in the other. The direction of the missing energy, being mainly that of the $Z^{*}$, is far from the quark jets. In contrast, in $\mathrm{e}^{+} \mathrm{e}^{-} \rightarrow \mathrm{q} \overline{\mathrm{q}}$ events (the main background), the two jets from the $q \bar{q}$ system are typically coplanar with the beam axis, seldom leading to low energy deposits in any one hemisphere. The relatively small missing energy is mostly due to the jet energy resolution or the undetected neutrinos within the jets. As a conse-

\#1 The L3 detector simulation is based on GEANT Version 3.14. See ref. [13]. The GHEISHA program [14] is used to simulate hadronic interactions. 
quence the missing energy direction is close to one of the jet axes.

The search for Higgs candidates is carried out taking advantage of these signatures.

In the preselection a set of cuts is applied to eliminate a large fraction of the background due to the $q \overline{\mathrm{q}}$, $\tau^{+} \tau^{-}$, two photon processes, cosmic rays and beam gas interactions. We require that:

- The invariant mass of the event calculated from all the calorimetric clusters (assumed to be massless), $M_{\text {vis }}$, is within the range $25-70 \mathrm{GeV}$.

- The energy imbalance transverse to the beam axis is larger than $15 \%$ and that parallel to the beam axis is less than $45 \%$ of the visible energy. The direction of the energy imbalance is more than 0.4 rad away from the beam axis.

- There are more than four charged tracks with transverse momenta larger than $0.3 \mathrm{GeV}$ and with distances of closest approach to the beam axis less than $5 \mathrm{~mm}$ and there are more than 15 calorimetric clusters.

The acceptance of the above cuts including trigger efficiency for $\mathrm{H}^{0} \nu \bar{\nu}$ events with $m_{\mathrm{H}}$ of $60 \mathrm{GeV}$ is $73 \%$ and we are left with $1.2 \%$ of the hadronic $Z$ decays, the background from all other sources being negligible.

For the final selection we use a set of cuts based on topological variables which are mostly related to the jet axis measurement since the jet directions are usually well defined even for events with large missing energy. The jet reconstruction starts by identifying the two calorimetric clusters that have the largest invariant mass. The plane perpendicular to that containing the direction vectors of the two clusters and bisecting the angle defined by them divides the event into two hemispheres. All the clusters in each hemisphere are combined to form a jet whose direction is determined by adding the momentum vectors of the clusters. Hence we obtain exactly two jets for each event, corresponding to the primary $q \bar{q}$ pair. The energies of both reconstructed jets are required to exceed $8 \mathrm{GeV}$. We define then a unit vector $\widehat{b}$ which is opposite to the sum of the unit vectors along the two jet directions.

Finally, we use the set of cuts from our previous publication [7]. An event is accepted if it satisfies the following criteria:

- $E_{90}<10 \mathrm{GeV}$ and $E_{60}<3 \mathrm{GeV}$, where $E_{90}$ and $E_{60}$ are the energies deposited in the cones with half opening angles of $90^{\circ}$ and $60^{\circ}$ respectively around $\widehat{b}$.
- The largest angular region, in the $r-\phi$ plane, where no tracks are present must be greater than $1.1 \mathrm{rad}$. - $E_{\mathrm{I}}<1 \mathrm{GeV}$, where $E_{\mathrm{I}}$ is the energy deposited in a cone of $20^{\circ}$ half opening angle around the missing energy direction.

$-\xi<160^{\circ}$ and $\zeta<170^{\circ}$, where $\xi$ and $\zeta$ are the angles between the two jets in space and in the $r-\phi$ plane respectively.

The above selection is sensitive only to the decays of the Higgs boson into q $\bar{q}$ pairs. In order to improve the selection efficiency by detecting decays $\mathrm{H}^{0} \rightarrow \tau^{+} \tau^{-}$ we alternatively use the following selection criteria:

- There are less than 18 calorimetric clusters and the total energy of all the calorimetric clusters is within the range $15-75 \mathrm{GeV}$.

- The energy imbalance transverse to the beam axis is larger than $5 \mathrm{GeV}$ and that parallel to the beam axis is less than $45 \%$ of the visible energy.

- There are 2 charged narrow jets. We define narrow jets as those which have the ratio of calorimetrical energies in $15^{\circ}$ and $30^{\circ}$ cones around their axes exceeding 0.9 .

- $E_{90}<3 \mathrm{GeV}$ and $E_{60}<1.5 \mathrm{GeV}$.

- The largest angular region in the $r-\phi$ plane where no tracks are present is greater than $3.0 \mathrm{rad}$.

- the angle between the two jets in the $r-\phi$ plane is less than $160^{\circ}$.

The overall detection efficiency for the Higgs signal as a function of the $\mathrm{H}^{0}$ mass is shown in table 1 .

The relative uncertainty in the selection efficiency has been found to be less than $1.5 \%$ for a $60 \mathrm{GeV}$ Higgs mass [7].

No candidates have been found in our data sample, and no events pass the selection from a sample of $1140000 \mathrm{q} \overline{\mathrm{q}}$ Monte Carlo [15] events.

\section{4. $\mathrm{H}^{0} \mathrm{e}^{+} \mathrm{e}^{-}$event selection}

The signature of this process is the presence of two energetic and well separated electrons coming from the virtual $\mathrm{Z}^{*}$ isolated from the $\mathrm{H}^{0}$ decay products. The main sources of background are the four fermion process $\mathrm{e}^{+} \mathrm{e}^{-} \rightarrow \mathrm{e}^{+} \mathrm{e}^{-} \mathrm{q} \overline{\mathrm{q}}[16]$ and the double semileptonic decay $\mathrm{Z} \rightarrow \mathrm{b} \bar{b} \rightarrow \mathrm{e}^{+} \mathrm{e}^{-} X$.

In our selection low multiplicity events, such as $\mathrm{e}^{+} \mathrm{e}^{-}$and $\tau^{+} \tau^{-}$final states, are removed by requiring at least 15 clusters in the electromagnetic calorimeter. 
Table 1

Selection efficiencies (in \%) for Higgs events in the different channels. The efficiencies for the $\mathrm{H}^{0} \nu \bar{\nu}$ and $\mathrm{H}^{0} \mu^{+} \mu^{-}$channels are the same for 1990 and 1991-1992 data.

\begin{tabular}{llllllll} 
Higgs mass (GeV) & 30 & 40 & 50 & 55 & 60 & 65 & 70 \\
$\mathrm{H}^{0} \nu \bar{\nu}$ channel & 29.5 & 52.8 & 57.7 & 51.8 & 46.2 & 28.0 & 15.0 \\
$\mathrm{H}^{0} \mathrm{e}^{+} \mathrm{e}^{-}$channel (1991-1992) & 58.2 & 55.2 & 52.2 & 50.5 & 49.4 & 47.8 & 44.0 \\
$\mathrm{H}^{0} \mathrm{e}^{+} \mathrm{e}^{-}$channel (1990) & 45.5 & 38.0 & 35.0 & 32.0 & 29.0 & 28.0 & 25.5 \\
$\mathrm{H}^{0} \mu^{+} \mu^{-}$channel & 52.6 & 54.6 & 53.4 & 49.0 & 47.6 & 41.6 & 37.0 \\
\hline
\end{tabular}

To reduce the hadronic background in our sample we require that the two most energetic clusters have energies greater than $3 \mathrm{GeV}$ and that the sum of their energies exceeds $15 \mathrm{GeV}$; in addition the opening angle between these two clusters must be larger than $40^{\circ}$.

The identification of electromagnetic particles is mainly based on the energy deposition pattern in the electromagnetic calorimeter. We consider the ratio of the energy deposited in a $3 \times 3$ crystal array and the energy deposited in a $5 \times 5$ array both centred on the most energetic crystal in the cluster. After applying a position-dependent leakage correction to both measurements the distribution of this ratio is approximately gaussian, centered at 1.0 with a width of $1 \%$. Electromagnetic candidates are identified by requiring that this ratio is less than $3 \sigma$ away from the above mean value.

The isolation of the electron candidates is further ensured by imposing the following conditions:

- The additional energy deposited in the electromagnetic calorimeter in a cone of $15^{\circ}$ half opening angle around the direction of the highest energy cluster must not exceed $5 \%$ of the cluster energy and the energy measured in the hadron calorimeter in the same cone must be less than $3 \mathrm{GeV}$.

- The additional energy deposited in the electromagnetic calorimeter in a cone of $15^{\circ}$ half opening angle around the second most energetic cluster must not exceed $7 \%$ of its energy.

To complete the identification of the electrons we consider all pairs out of three most energetic electron candidates. The most energetic cluster in the pair is required to match in azimuthal angle with exactly one track and the second most energetic cluster with at least one track. For both clusters the matching has to be within a $4 \sigma$ cut where $\sigma$ depends on the energies and on the polar angles of the clusters.

To identify the Higgs boson decay products we ex- amine the non-electron jets in the event. Indicating with $P_{\perp}$ the transverse momentum of each electron with respect to the nearest jet, we require the sum of the two $P_{\perp}$ to be larger than $10 \mathrm{GeV}$.

To reject a background from the four fermion process we require that

$2 M_{\mathrm{e}^{+} \mathrm{e}^{-}}+M_{\text {miss }}>80 \mathrm{GeV}$

where $M_{\mathrm{e}^{+} \mathrm{e}^{-}}$is the invariant mass of the electron pair and $M_{\text {miss }}$ is the missing mass with respect to these electrons. Figure 1 shows the effect of this cut on the remaining candidates as well as on the expected Higgs signal after all above cuts are applied.

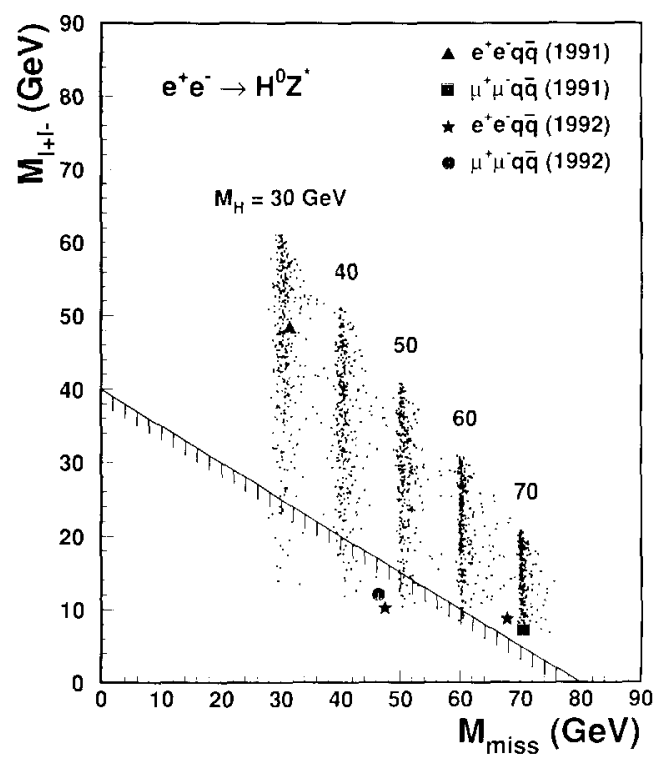

Fig. 1. The mass of the dilepton pair $M_{\ell+\ell^{-}}$plotted against the missing mass to the same pair $M_{\text {miss }}$ after all other cuts are applied. The remaining candidates together with the expected signal from 30, 40, 50, 60 and $70 \mathrm{GeV}$ Higgs bosons are shown. The line represents the $2 M_{\ell+\ell^{-}}+M_{\text {miss }}>80 \mathrm{GeV}$ cut described in the text. 


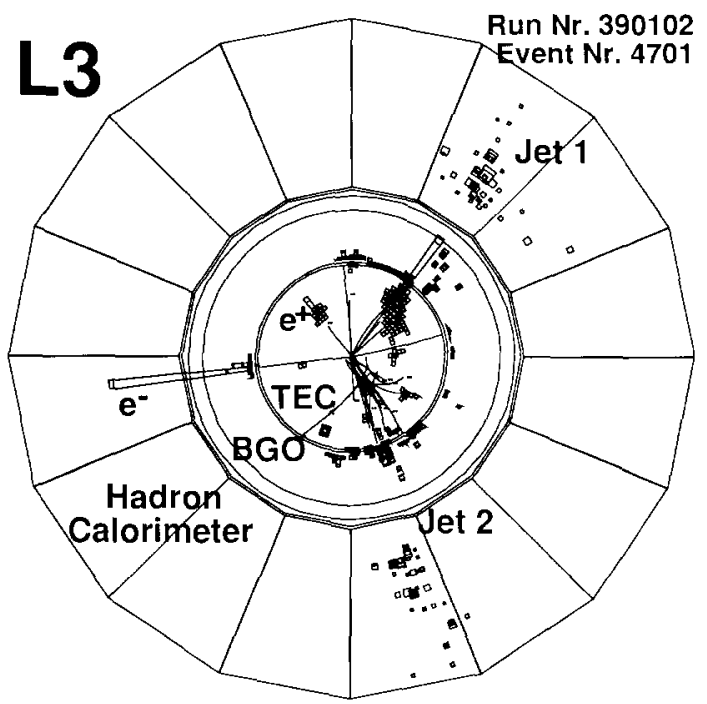

Fig. 2. The $67.6 \mathrm{GeV}$ mass candidate found in 1992 in the $\mathrm{H}^{0} \mathrm{e}^{+} \mathrm{e}^{-}$channel shown in the plane perpendicular to the beam line. The lines in the TEC represent the reconstructed charged tracks. The size of the symbols indicating individual calorimetric hits (towers in the electromagnetic calorimeter and boxes in the hadron calorimeter) corresponds to the energy deposition of that hit. The towers which appear in the TEC region in this projection belong to the $\mathrm{BGO}$ endcaps.

The selection efficiencies for the signal are shown in table 1 for the 1990 and 1991-1992 setups. The efficiency for the 1990 data is lower due to the lower geometrical acceptance of the BGO calorimeter which did not include the endcaps.

Two events passed the above selection criteria. The first one, with the missing mass to two electrons of $31.4 \pm 1.5 \mathrm{GeV}$, is from the 1991 data sample [7]; the second one, from the 1992 data sample, has the missing mass recoiling against the electron pair of $67.6 \pm 0.7 \mathrm{GeV}$. This event is shown in fig. 2 and its main parameters are given in table 2 . Both events are consistent with the background process $\mathrm{e}^{+} \mathrm{e}^{-} \rightarrow$ $\mathrm{e}^{+} \mathrm{e}^{-} \mathrm{q} \overrightarrow{\mathrm{q}}$ from which we expect $2.5 \pm 0.4$ events. The background from other processes is negligible.

\section{5. $\mathbf{H}^{0} \mu^{+} \mu^{-}$event selection}

This analysis is based on the selection of events with well isolated muons together with other charged
Table 2

The energies $(E)$, the polar angles with respect to the beam line $(\theta)$ and the azimuthal angles $(\phi)$ of the main constituents of the $1992 \mathrm{H}^{0} \mathrm{e}^{+} \mathrm{e}^{-}$candidate are indicated. The main parameters of the event are: a visible energy of $109.0 \pm 12.5 \mathrm{GeV}$, a missing mass recoiling against the final state electrons of $67.6 \pm 0.7 \mathrm{GeV}$, an invariant mass of the electron-positron pair of $8.0 \pm 0.3 \mathrm{GeV}$ and a measured mass of the hadronic system of $82.2 \pm 12.3 \mathrm{GeV}$.

\begin{tabular}{rrrr} 
& $E(\mathrm{GeV})$ & $\theta(\mathrm{deg})$. & $\phi($ deg. $)$ \\
\hline jet $_{1}$ & $58.3 \pm 8.9$ & $152.3 \pm 2.1$ & $52.9 \pm 2.1$ \\
jet $_{2}$ & $30.8 \pm 5.5$ & $36.6 \pm 2.1$ & $284.6 \pm 2.1$ \\
$\mathrm{e}^{+}$ & $4.8 \pm 0.1$ & $20.7 \pm 0.2$ & $127.7 \pm 0.2$ \\
$\mathrm{e}^{-}$ & $16.2 \pm 0.2$ & $63.4 \pm 0.1$ & $186.1 \pm 0.3$ \\
\hline
\end{tabular}

particles present in the fragmentation of the heavy quark pair from the Higgs decay.

Muons are identified as tracks in the muon spectrometer that when extrapolated back towards the beam line pass within $3.5 \sigma$ from the interaction point, both in the $r-\phi$ and $z$ directions; in the following we refer to these tracks simply as muons.

We require the presence of at least one muon and at least 5 other tracks reconstructed in the TEC in order to remove cosmic ray, $\mu^{+} \mu^{-}, \tau^{+} \tau^{-}, \mathrm{e}^{+} \mathrm{e}^{-} \mu^{+} \mu^{-}$and $\pi^{+} \pi^{+} \mu^{+} \mu^{-}$events. In order to reduce the background from $\mathrm{Z} \rightarrow \mathrm{q} \overline{\mathrm{q}}$ events, we require the event thrust to be less than 0.92 .

To further reduce the background from the semileptonic decays of hadrons two sets of cuts are applied: set (i) to recognize a single well isolated muon and set (ii) to select the muon pair from the $Z^{*}$ decay. An event is accepted as a candidate if it has at least one muon satisfying the first set of cuts (i) or at least a pair of muons satisfying the second set of cuts (ii). This allows us to recover the events in which one of the two muons coming from the decay of the virtual $Z^{*}$ is not detected in the muon spectrometer.

To measure the isolation of a muon we define three quantities.

- $\mathcal{D}_{\mathrm{i}}=\left(E_{\mathrm{jet}_{i}}-p_{\mu_{i}}\right) / p_{\mu_{i}}$, where $E_{\mathrm{jet}_{i}}$ is the energy of the jet which includes the $i$ th muon and $p_{\mu_{i}}$ is the value of the muon momentum.

- $\mathcal{E}_{i}^{\mathrm{e}}$ and $\mathcal{E}_{i}^{\mathrm{h}}$ are the differences of the energies deposited in $30^{\circ}$ and $3^{\circ}$ cones around the $i$ th muon in the $\mathrm{BGO}$ and hadron calorimeters respectively. The subtraction diminishes the sensitivity of this variable 
to radiative photons.

The two sets of cuts are:

\section{Set (i)}

The muon must have a momentum larger than 10 $\mathrm{GeV}$. To ensure the isolation, we require less than 5 calorimetric clusters and at most one additional charged track, which must be isolated by more than $0.1 \mathrm{rad}$, in the jet which includes the muon. The isolation variable $\mathcal{D}$ must have a value of less than 0.3 , and the variable $\mathcal{E}^{\mathrm{e}}$ must be less than $2 \mathrm{GeV}$. In addition the missing energy of the event is required to be at least $10 \mathrm{GeV}$ due to the undetected muon.

\section{Set (ii)}

The opposite-sign muon pair is identified as being from the decay of the $Z^{*}$ if it satisfies the following criteria:

- There is at least one muon with less than 5 charged tracks in the associated jet.

- $\mathcal{D}_{1} \cdot \mathcal{D}_{2}<0.7$.

$-\min \left(\mathcal{E}_{1}^{\mathrm{e}}, \mathcal{E}_{2}^{\mathrm{e}}\right)<250 \mathrm{MeV}$.

$-\min \left(\mathcal{E}_{1}^{\mathrm{h}}, \mathcal{E}_{2}^{\mathrm{h}}\right)<1 \mathrm{GeV}$.

$-\min \left(p_{\mu_{1}}, p_{\mu_{2}}\right)>3.4 \mathrm{GeV}$ and $p_{\mu_{1}}+p_{\mu_{2}}>15 \mathrm{GeV}$.

In order to suppress the background from four fermion processes we use the same final cut as in the $\mathbf{H}^{0} \mathrm{e}^{+} \mathrm{e}^{-}$selection:

$2 M_{\mu^{+} \mu^{-}}+M_{\text {miss }}>80 \mathrm{GeV}$

For events passing selection (i) we compute $M_{\mu+\mu}$ and $M_{\text {miss }}$ in the following way: if there is a TEC track pointing to a calorimetric cluster compatible with a minimum ionizing particle, the undetected muon momentum vector is assumed to be the one measured by the TEC; if there is no such track, the undetected muon momentum vector is assumed to be the missing momentum vector of the event.

The Higgs selection efficiencies are shown in table 1.

One event with $M_{\text {miss }}=70.4 \pm 0.7 \mathrm{GeV}$ from the 1991 data sample passed the above selection criteria [7]. The expected background is computed to be $1.2 \pm 0.2$ events.

\section{Systematic uncertainties}

The sources of systematic errors on the number of expected Higgs events are the following:

- Theoretical uncertainty of less than $1 \%$ on the ratio between the Higgs boson production cross section and the $\mathrm{e}^{+} \mathrm{e}^{-} \rightarrow \mathrm{q} \overline{\mathrm{q}}$ cross section [17].

- Experimental uncertainty of $0.5 \%$ on the corrected number of hadronic $Z$ decays used for the normalization.

- Theoretical uncertainty on the Higgs decay branching ratios which contributes an error of $0.7 \%$ to the detection efficiency [5].

- Error on the Higgs detection efficiency of $1.5 \%$ due to the uncertainties in the fragmentation model and due to the uncertainties in the detector calibration constants [7].

- Error on the Higgs detection efficiency of $1.5 \%$ due to Monte Carlo statistics.

Combining these errors in quadrature we obtain a systematic uncertainty of $2.5 \%$.

\section{Mass limit}

We have observed two $\mathrm{e}^{+} \mathrm{e}^{-}$hadrons and one $\mu^{+} \mu^{-}$hadrons events that pass our selection criteria. From Monte Carlo studies, we expect the number of background events to be $2.5 \pm 0.4 \mathrm{e}^{+} \mathrm{e}^{-} \mathrm{q} \overline{\mathrm{q}}$ for the $\mathrm{H}^{0} \mathrm{e}^{+} \mathrm{e}^{-}$channel and $1.2 \pm 0.2 \mu^{+} \mu^{-} \mathrm{q} \overline{\mathrm{q}}$ for the $\mathrm{H}^{0} \mu^{+} \mu^{-}$channel. Taking into account the mass resolution of the L3 detector we conclude that none of these events is consistent with a Higgs boson mass in the vicinity of $60 \mathrm{GeV}$. Therefore we take the $95 \%$ confidence level limit on the Higgs boson mass corresponding to three events. Taking into account the $2.5 \%$ systematic error on the number of expected events, we obtain a $95 \%$ C.L. lower limit on the mass of the Higgs boson of $57.7 \mathrm{GeV}$.

Figure 3 shows the number of expected Higgs events in the mass range from 30 to $70 \mathrm{GeV}$. The $95 \%$ C.L. line, also shown in the figure, was obtained with the likelihood function of the candidates, taking into account the number of expected events from the background and the mass measurement errors. 


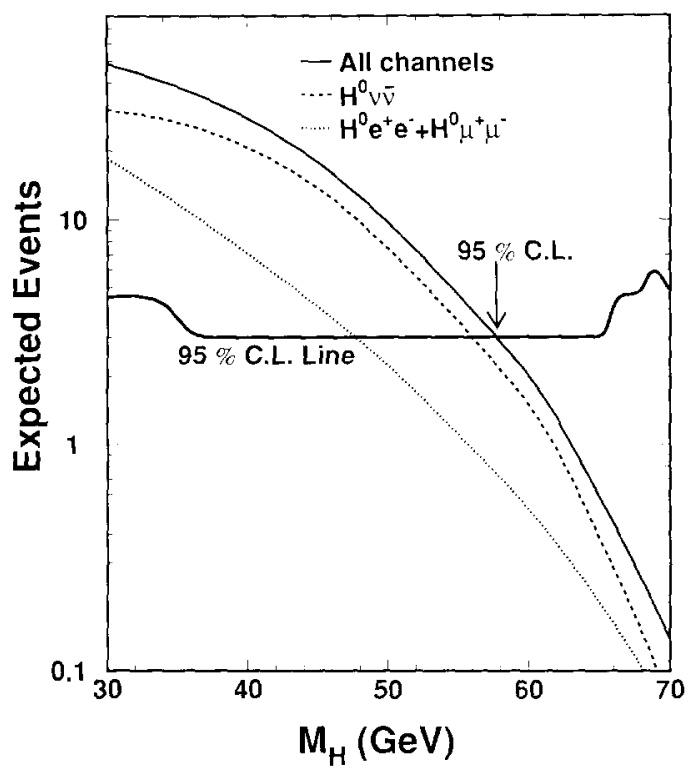

Fig. 3. Number of Higgs events expected in the different channels. The $95 \%$ confidence level line is shown and the Higgs mass limit at the $95 \%$ C.L. is indicated.

\section{Acknowledgement}

We wish to express our gratitude to the CERN accelerator divisions for the excellent performance of the LEP machine. We acknowledge the efforts of all engineers and technicians who have participated in the construction and maintenance of this experiment.

\section{References}

[1] S.L. Glashow, Nucl. Phys. 22 (1961) 579; S. Weinberg, Phys. Rev. Lett. 19 (1967) 1264; A. Salam, Elementary particle theory, ed. N. Svartholm (Almquist and Wiksell, Stockholm, 1968) p. 367.

[2] P.W. Higgs, Phys. Lett. 12 (1964) 132; Phys, Rev, Lett. 13 (1964) 508; Phys. Rev. 145 (1966) 1156 ;

F. Englert and R. Brout, Phys. Rev. Lett. 13 (1964) 321.

[3] B.L. Ioffe and V.A. Khoze, What can be expected from experiments on colliding $\mathrm{e}^{+} \mathrm{e}^{-}$beams with $\approx 100 \mathrm{GeV}$, preprint LINP, Leningrad (November 1976);
J.D. Bjorken, in: Proc. 1976 SLAC Summer Institute on Particle physics (Stanford), ed. M.C. Zipf (Stanford Linear Accelerator Center, Stanford, CA, 1977) p. 1; J.Finjord, Phys. Scripta 21 (1980) 143.

[4] P.J. Franzini et al. in: Z Physics at LEP1, CERN Report CERN-89-08, eds. G. Altarelli, R. Kleiss and C. Verzegnassi (CERN, Geneva, 1989) Vol. 2, p. 59 and references therein.

[5] E. Braaten and J.P. Leveille, Phys. Rev. D 22 (1980) 715.

[6] L3 Collab., B. Adeva et al., Phys. Lett. B 248 (1990) 203; B 252 (1990) 518; B 257 (1991) 450;

L3 Collab., O. Adriani et al,, Phys. Lett. B 294 (1992) 457; Preprint CERN-PPE/92-163 (1992), to be published in Z. Phys. C.

[7] L3 Collab., B. Adeva et al., Phys. Lett. B 283 (1992) 454.

[8] ALEPH Collab., D.Decamp et al., Phys. Lett. B 236 (1990) 233; B 241 (1990) 141; B 245 (1990) 289; B 246 (1990) 306; Phys. Rep. 216 (1992) 253.

[9] DELPHI Collab., P.Abreu et al., Nucl. Phys. B 342 (1990) 1; Z. Phys. C 51 (1991) 25; Nucl. Phys. B 373 (1992) 3.

[10] OPAL Collab., M.Z. Akrawy et al., Phys. Lett. B 236 (1990) 224, Z. Phys. C 49 (1990) 1, Phys. Lett. B 251 (1990) 211; B 253 (1991) 511;

OPAL Collab., P.D. Acton et al., Phys. Lett, B 268 (1991) 122.

[11] L3 Collab., B. Adeva et al., Nucl. Instrum. Methods A 289 (1990) 35.

[12] O. Adriani et al., Nucl. Instrum. Methods A 302 (1991) 53.

[13] R. Brun et al., GEANT 3, CERN DD/EE/84-1 (Revised), September 1987.

[14] H. Fesefeldt, RWTH Aachen Preprint PITHA 85/02 (1985).

[15] T. Sjöstrand and M. Bengtsson, Comput. Phys. Commun. 43 (1987) 367;

T. Sjöstrand, in: $Z$ Physics at LEP1, CERN Report CERN-89-08, Vol. 3, p. 143.

[16] E.W.N. Glover, R. Kleiss and J.J. van der Bij, Z. Phys. C 47 (1990) 435;

J. Hilgart, R. Kleiss and F. Le Diberder, Comput. Phys. Commun. 75 (1993) 191.

[17] M. Consoli and W. Hollik, in: Z Physics at LEP1, CERN Report CERN-89-08, eds. G. Altarelli, R. Kleiss and C. Verzegnassi (CERN, Geneva, 1989) Vol. 1, p. 39; B.A. Kniehl, Radiative corrections to Higgs production from Z decay, preprint DESY 92-021, February 1992; F.A. Berends, W.L. van Neerven and G.J.H. Burgers, Nucl. Phys. B 297 (1988) 429. 